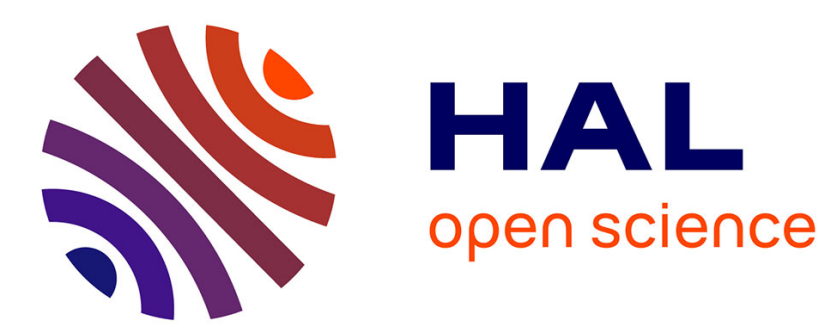

\title{
Homogenisation of advective-diffusive transport in poroelastic media
}

Pascale Royer, Fabien Cherblanc

\section{To cite this version:}

Pascale Royer, Fabien Cherblanc. Homogenisation of advective-diffusive transport in poroelastic media. Mechanics Research Communications, 2010, 37 (2), pp.133 - 136. 10.1016/j.mechrescom.2009.11.003 . hal-01512642

\section{HAL Id: hal-01512642 \\ https://hal.science/hal-01512642}

Submitted on 24 Apr 2017

HAL is a multi-disciplinary open access archive for the deposit and dissemination of scientific research documents, whether they are published or not. The documents may come from teaching and research institutions in France or abroad, or from public or private research centers.
L'archive ouverte pluridisciplinaire HAL, est destinée au dépôt et à la diffusion de documents scientifiques de niveau recherche, publiés ou non, émanant des établissements d'enseignement et de recherche français ou étrangers, des laboratoires publics ou privés. 


\title{
Homogenisation of advective-diffusive transport in poroelastic media
}

\author{
P. Royer, F. Cherblanc \\ Laboratoire de Mécanique et Génie Civil, Université Montpellier 2, CNRS \\ Place Eugène Bataillon, 34095 Montpellier, France \\ pascale.royer@univ-montp2.fr
}

June 9, 2011

\begin{abstract}
Advective-diffusive solute transport in a poroelastic medium is usually modelled at the macroscopic scale by considering a poro-elastic formulation associated with the classical advective-diffusive solute transport equation. The purpose of the present work is to rigorously determine the macroscopic model by starting with the pore-scale equations that describe transient advective/diffusive solute transport in a saturated deformable elastic porous medium. The macroscopic model is derived by using an homogenisation method. It includes Biot's model of consolidation and a transport equation of advective-diffusive type. This latter contains a coupling term which reveals a mechanically-induced solute transport mechanism.
\end{abstract}

\section{Introduction}

When solute transport occurs in a deformable porous media, some coupling effects are observed which are generally reported as "mechanically-induced solute transport" or "activated solute transport". Indeed, the consolidation of a saturated porous medium produces fluid flow which participates to the solute convective transport. These issues have been theoretically examined in the context of soil contaminant migration and particularly in capped waste deposits (Brannon and Poitdexter-Rollings, 1990; Alshawabkeh et al., 2005; Arega and Hayter, 2008). Various consolidation and contaminant transport models have been developped to capture some non-linear effects resulting from a large strain formulation (Smith, 2000; Peters and Smith, 2002; Fox, 2007).

To get further insight into mechanically-induced solute transport, cyclic loading is applied on saturated deformable porous media as it is known to enhance solute transport mechanism (Tate, 2001; Mauck et al., 2003). This coupling effect is of major interest when dealing for instance with the design of drug delivery system (Urciuolo et al., 2008). This transport enhancement mechanism 
also participates in the cell nutrients transfer through avascular biological tissue such as articular cartilage or intervertebral disk (O'Hara et al., 1990; Evans and Quinn, 2006). Some attempts to develop a theroretical solute transport model a the scale of a canaliculi have been proposed (Wang et al., 2000; Goldsztein and Santamarina, 2004).

Several experimental and numerical works have focused on the identification of the main parameters (frequency, amplitude, ...) that govern this mechanism (Sengers et al., 2004; Urciuolo et al., 2008). These models are usually based on a poro-elastic formulation associated with the classical advective-diffusive transport equation. Both models are written directly at the macroscopic scale without wondering if coupling effects between solute transport and mechanical strains could affect these classical macroscopic equations.

In the present work, we propose to revisit this issue by means of a theoretical upscaling procedure to rigorously determine the macroscopic model by starting with the pore-scale equations that describe transient advective/diffusive solute transport in a saturated deformable elastic porous medium. To upscale the pore-scale description, we use the homogenisation method for periodic structures, also called homogenisation method of multiple scales asymptotic expansions (Sanchez-Palencia, 1980) and the methodology introduced in Auriault (1991) is applied. The macroscopic behaviour which is thus derived includes Biot's model of consolidation and an advective-diffusive transport equation. This latter contains a coupling term which is emerging from the scale transition and characterizes a mechanically-induced solute transport mechanism.

\section{Homogenisation method}

\subsection{Medium under consideration}

The method of multiple scales is based upon the fundamental assumption of separation of scales. The condition of periodicity is also required, which has no impact upon the form of the macroscopic description. We thus consider a periodic porous medium, of macroscopic characteristic size $L$, and made of a solid matrix and of a fluid-saturated pore space. We further denote the periodic cell by $\Omega$, its characteristic length by $l$, and we formulate the condition of separation of scales by $\varepsilon=l / L \ll 1$. Within the periodic cell, we denote by $\Omega_{\mathrm{f}}$ the fluid-saturated pore domain, by $\Omega_{\mathrm{s}}$ the solid matrix, and by $\Gamma$ their common interface, as depicted in Fig. 1 . Using the two characteristic length, $l$ and $L$, and the physical space variable, $\vec{X}$, we define two dimensionless space variables: $\vec{y}=$ $\vec{X} / l, \vec{x}=\vec{X} / L$. Since the condition of separation of scales $(\varepsilon \ll 1)$ is supposed to be satisfied, then variables $\vec{y}$ and $\vec{x}$ can be considered as two independent space variables: $\vec{y}$ describes the local scale, while $\vec{x}$ is the macroscopic space variable. As a consequence, the unknown fields (stress tensors, displacements, fluid velocity and pressure, ...) are, a priori, functions of both space variables $\vec{y}$ and $\vec{x}$. Invoking the differentiation rule for multiple variables, the gradient 


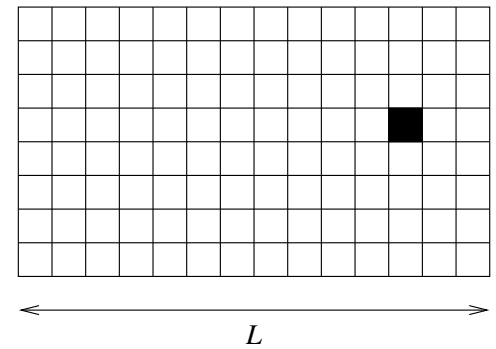

(a)

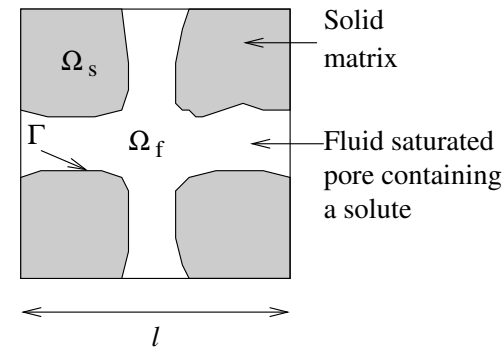

(b)

Figure 1: Porous medium : (a) Macroscopic sample; (b) Periodic unit cell.

operator with respect to the physical variable, $\vec{X}$, is written as

$$
\nabla_{X}=\frac{1}{l} \nabla_{y}+\frac{1}{L} \nabla_{x}
$$

\subsection{Dimensionless pore-scale description}

The methodology introduced in Auriault (1991) firstly consists in writing the dimensionless pore-scale description, which is the set of dimensionless equations that describe the phenomena being considered within the periodic unit cell depicted in Fig. 1. Each quantity in a dimensionless equation is the ratio of its physical counterpart to its characteristic value (indexed by ${ }^{*}$ ). This writing gives rise to dimensionless numbers, which are defined by means of characteristic values. We consider the elasticity equations in the solid domain $\Omega_{\mathrm{s}}$, the equations of flow for a Stokesian fluid and the convection-diffusion equation for the solute in the fluid-saturated pore domain $\Omega_{\mathrm{f}}$, together with the boundary conditions over the interface $\Gamma$. The dimensionless writing of the equations requires the choice of a characteristic length for the dimensionless writing of space derivatives. We arbitrarily choose $L$ as the reference characteristic length. The dimensionless gradient operator is thus given by

$$
\nabla=L \nabla_{X}=\varepsilon^{-1} \nabla_{y}+\nabla_{x}
$$

The dimensionless pore-scale description being considered is given by the equations presented below.

Equations in the solid domain $\Omega_{\mathrm{s}}$

$$
\begin{array}{r}
\nabla \cdot \sigma_{\mathrm{s}}=\overrightarrow{0}, \\
\sigma_{\mathrm{s}}=\mathbf{a}: \mathbf{e}\left(\vec{u}_{\mathrm{s}}\right) .
\end{array}
$$


Equations of fluid motion in $\Omega_{\mathrm{f}}$

$$
\begin{array}{r}
\mathbb{F}_{\mathrm{L}} \mu \Delta \vec{v}_{\mathrm{f}}-\nabla p_{\mathrm{f}}=\overrightarrow{0}, \\
\sigma_{\mathrm{f}}=-p_{\mathrm{f}}+\mathbb{F}_{\mathrm{L}} 2 \mu \mathbf{D}\left(\vec{v}_{\mathrm{f}}\right), \\
\nabla \cdot \vec{v}_{\mathrm{f}}=0,
\end{array}
$$

where $\mathbb{F}_{\mathrm{L}}$ is a dimensionless number, defined by $\mathbb{F}_{\mathrm{L}}=\frac{\mu^{*} v_{\mathrm{f}}^{*}}{p_{\mathrm{f}}^{*} L}$.

Advective-diffusive solute transport in $\Omega_{\mathrm{f}}$

$$
\mathbb{N}_{\mathrm{L}} \frac{\partial c}{\partial t}+\mathbb{P} e_{\mathrm{L}} \nabla \cdot\left(\vec{v}_{\mathrm{f}} c\right)=D \Delta c
$$

where the dimensionless numbers are defined by $\mathbb{N}_{\mathrm{L}}=\frac{L^{2}}{D^{*} t}$ and $\mathbb{P} e_{\mathrm{L}}=\frac{v_{\mathrm{f}}^{*} L}{D^{*}}$.

Conditions over the interface $\Gamma$

$$
\begin{array}{r}
\sigma_{\mathrm{f}} \cdot \vec{n}=\sigma_{\mathrm{s}} \cdot \vec{n}, \\
\vec{v}_{\mathrm{f}}=\frac{\partial \vec{u}_{\mathrm{s}}}{\partial t}, \\
\nabla c \cdot \vec{n}=0 .
\end{array}
$$

\subsection{Orders-of-magnitude of dimensionless numbers}

The methodology introduced in Auriault (1991) consists in estimating the dimensionless numbers with respect to $\varepsilon$. As we are interested in the case which leads to a diphasic macroscopic behaviour for the fluid/solid system, the case where fluid motion is generated must be considered, which corresponds to the order (Auriault, 1991) $\mathbb{F}_{\mathrm{L}}=\mathcal{O}\left(\varepsilon^{2}\right)$. It has been shown in Auriault and Adler (1995) that in rigid porous media, a transient advective-diffusive macroscopic solute transport is obtained when $\mathbb{N}_{\mathrm{L}}=\mathcal{O}\left(\varepsilon^{0}\right)$ and $\mathbb{P} e_{\mathrm{L}}=\mathcal{O}\left(\varepsilon^{0}\right)$.

\subsection{Homogenisation procedure}

The homogenisation method being used is an asymptotic approach. It is therefore based upon the fundamental statement that the unknown fields can be written in the form of asymptotic expansions in powers of $\varepsilon$

$$
\psi=\psi^{(0)}(\vec{y}, \vec{x})+\varepsilon \psi^{(1)}(\vec{y}, \vec{x})+\varepsilon^{2} \psi^{(2)}(\vec{y}, \vec{x})+\ldots
$$

in which functions $\psi^{(i)}$ are $\Omega$-periodic in variable $\vec{y}$, with $\psi=\boldsymbol{\sigma}_{\mathrm{s}}, \boldsymbol{\sigma}_{\mathrm{f}}, p_{\mathrm{f}}, c, \vec{v}_{\mathrm{f}}, \vec{u}_{\mathrm{s}}$. The method consists in incorporating the asymptotic expansions in the dimensionless local description (Eqs. 3-11), while replacing the dimensionless numbers by their orders of magnitude in power of $\varepsilon$ and taking into account the expression of the dimensionless gradient operator (Eq. 2). This leads to approximated governing equations and boundary conditions at the successive orders, which together with the condition of periodicity define well posed boundary-value problems in the periodic unit cell. Existence of solutions requires that volume averaged equations be satisfied. These latter actually describe the macroscopic behaviour at successive orders. 


\section{Macroscopic poroelastic model}

Homogenisation of the fluid/solid equations (Eqs. 3-7, 9 and 10) has been performed by Auriault and Sanchez-Palencia (1977). It leads to the following macroscopic model, which is identical to Biot's model of poroelasticity

$$
\begin{aligned}
\nabla \cdot<\boldsymbol{\sigma}^{(0)}> & =\overrightarrow{0} \\
<\boldsymbol{\sigma}^{(0)}> & =\mathbf{C}: \mathbf{e}\left(\vec{u}_{\mathrm{s}}^{(0)}\right)-\boldsymbol{\alpha} p_{\mathrm{f}}^{(0)}, \\
\nabla_{x} \cdot\left(<\vec{v}_{\mathrm{f}}^{(0)}>^{\mathrm{f}}-\phi \frac{\partial \vec{u}_{\mathrm{s}}^{(0)}}{\partial t}\right) & =\boldsymbol{\alpha} \otimes \mathbf{e}\left(\vec{u}_{\mathrm{s}}^{(0)}\right)-\beta \frac{\partial p_{\mathrm{f}}^{(0)}}{\partial t}, \\
<\vec{v}_{\mathrm{f}}^{(0)}>^{\mathrm{f}}-\phi \frac{\partial \vec{u}_{\mathrm{s}}^{(0)}}{\partial t} & =-\frac{\mathbf{K}}{\mu} \nabla p_{\mathrm{f}}^{(0)} .
\end{aligned}
$$

In the above equations $\left\langle\boldsymbol{\sigma}^{(0)}>\right.$ is the first-order averaged total stress tensor, $\mathbf{C}$ is the effective elastic tensor, while $\boldsymbol{\alpha}$ represents the elasticity coefficient of Biot, $\beta$ is the medium compressibility and $\mathbf{K}$ denotes the permeability tensor. The term $\left\langle\vec{v}_{\mathrm{f}}^{(0)}>^{\mathrm{f}}-\phi \partial \vec{u}_{\mathrm{s}}^{(0)} / \partial t\right.$ where $\vec{u}_{\mathrm{s}}^{(0)}=\vec{u}_{\mathrm{s}}^{(0)}(\vec{x}, t)$ represents the fluid relative velocity and the porosity $\phi$ is defined by $\phi=\left|\Omega_{\mathrm{f}}\right| /|\Omega|$. The distinct averages being introduced are defined by:

$$
<\psi>=<\psi>^{\mathrm{s}}+<\psi>^{\mathrm{f}},
$$

where

$$
<\psi>^{\mathrm{s}}=\frac{1}{|\Omega|} \int_{\Omega_{\mathrm{s}}} \psi d \Omega, \quad<\psi>^{\mathrm{f}}=\frac{1}{|\Omega|} \int_{\Omega_{\mathrm{f}}} \psi d \Omega .
$$

Details on the derivation of the above model can be found in Auriault and Sanchez-Palencia (1977).

\section{Homogenisation of transport equations}

Determination of $c^{(0)}$ and $c^{(1)}$

At the first two orders, we get boundary-value problems which are identical to those obtained in a rigid porous medium (Auriault and Adler, 1995). They lead to

$$
\begin{aligned}
& c^{(0)}=c^{(0)}(\vec{x}, t), \\
& c^{(1)}=\vec{\chi} \cdot \nabla_{x} c^{(0)}+\bar{c}^{(1)}(\vec{x}, t),
\end{aligned}
$$

where $\bar{c}^{(1)}$ is an arbitrary function and where $\vec{\chi}$ is defined by the following local problem to be solved on the unit cell:

$$
\left\{\begin{array}{rll}
\nabla_{y} \cdot\left(D\left(\nabla_{y} \vec{\chi}+\mathbf{I}\right)\right) & =\overrightarrow{0} \quad \text { in } \Omega_{\mathrm{f}}, \\
\left(\nabla_{y} \vec{\chi}+\mathbf{I}\right) \cdot \vec{n} & =\overrightarrow{0} \quad \text { on } \Gamma, \\
<\vec{\chi}>^{\mathrm{f}} & =\overrightarrow{0}, & \text { and } \quad \vec{\chi} \text { is } \Omega \text {-periodic. }
\end{array}\right.
$$


Determination of the first-order macroscopic description

At the third-order, transport equations lead to the following system:

$$
\begin{array}{rr}
\frac{\partial c^{(0)}}{\partial t}-\nabla_{y} \cdot( & \left.D\left(\nabla_{y} c^{(2)}+\nabla_{x} c^{(1)}\right)-c^{(0)} \vec{v}_{\mathrm{f}}^{(1)}-c^{(1)} \vec{v}_{\mathrm{f}}^{(0)}\right)- \\
-\nabla_{x} \cdot\left(D\left(\nabla_{y} c^{(1)}+\nabla_{x} c^{(0)}\right)-c^{(0)} \vec{v}_{\mathrm{f}}^{(0)}\right)=0 & \text { in } \Omega_{\mathrm{f}}, \\
\left(\nabla_{y} c^{(2)}+\nabla_{x} c^{(1)}\right) \cdot \vec{n}=0 & \text { on } \Gamma, \\
\vec{v}_{\mathrm{f}}^{(0)}=\frac{\partial \vec{u}_{\mathrm{s}}^{(0)}}{\partial t}(\vec{x}, t) & \text { on } \Gamma .
\end{array}
$$

Integrating equation (Eq. 22) over the period, while taking the associated boundary-conditions (Eqs. 23 and 24) into account together with the condition of periodicity yields the first-order macroscopic transport equation. We firstly note that from the second-order expansion of (Eq. 7) and from (Eq. 19), we have:

$$
\nabla_{y} \cdot\left(c^{(0)} \vec{v}_{\mathrm{f}}^{(1)}\right)=c^{(0)} \nabla_{y} \cdot \vec{v}_{\mathrm{f}}^{(1)}=-c^{(0)} \nabla_{x} \cdot \vec{v}_{\mathrm{f}}^{(0)} .
$$

Integrating the thus transformed transport equation (Eq. 22), we obtain:

$$
\begin{aligned}
\phi \frac{\partial c^{(0)}}{\partial t}-\nabla_{x} & \cdot\left(\mathbf{D}^{\mathrm{eff}} \nabla_{x} c^{(0)}\right)+\nabla_{x} c^{(0)} \cdot\left(<\vec{v}_{\mathrm{f}}^{(0)}>^{\mathrm{f}}-\phi \frac{\partial \vec{u}_{\mathrm{s}}^{(0)}}{\partial t}\right)= \\
& -\phi \nabla_{x} c^{(0)} \cdot \frac{\partial \vec{u}_{\mathrm{s}}^{(0)}}{\partial t}-\frac{1}{|\Omega|} \int_{\Omega_{\mathrm{f}}} \nabla_{y} \cdot\left(c^{(1)} \frac{\partial \vec{u}_{\mathrm{s}}^{(0)}}{\partial t}\right) d \Omega
\end{aligned}
$$

in which $\mathbf{D}^{\text {eff }}$ represents the effective tensor of diffusion, and is defined by

$$
\mathbf{D}^{\text {eff }}=D \mathbf{T}, \quad \text { with } \quad \mathbf{T}=\frac{1}{|\Omega|} \int_{\Omega_{\mathrm{f}}}\left(\nabla_{y} \vec{\chi}+\mathbf{I}\right) d \Omega,
$$

where $\mathbf{T}$ denotes the tortuosity tensor (Quintard, 1993; Dormieux and Lemarchand, 2000). Now, using the expression of $c^{(1)}$ (Eq. 20), we get

$$
\begin{array}{r}
-\phi \nabla_{x} c^{(0)} \cdot \frac{\partial \vec{u}_{\mathrm{s}}^{(0)}}{\partial t}-\frac{1}{|\Omega|} \int_{\Omega_{\mathrm{f}}} \nabla_{y} \cdot\left(c^{(1)} \frac{\partial \vec{u}_{\mathrm{s}}^{(0)}}{\partial t}\right) d \Omega= \\
\frac{\partial \vec{u}_{\mathrm{s}}^{(0)}}{\partial t} \cdot\left\langle\nabla_{y} \vec{\chi}+\mathbf{I}>^{\mathrm{f}} \nabla_{x} c^{(0)}=\frac{\partial \vec{u}_{\mathrm{s}}^{(0)}}{\partial t} \cdot \mathbf{T} \nabla_{x} c^{(0)} .\right.
\end{array}
$$

The first-order macroscopic transport equation finally reads

$$
\begin{aligned}
& \phi \frac{\partial c^{(0)}}{\partial t}-\nabla_{x} \cdot\left(\mathbf{D}^{\text {eff }} \nabla_{x} c^{(0)}\right)+\left(<\vec{v}_{\mathrm{f}}^{(0)}>^{\mathrm{f}}-\phi \frac{\partial \vec{u}_{\mathrm{s}}^{(0)}}{\partial t}\right) \cdot \nabla_{x} c^{(0)} \\
& +\frac{\partial \vec{u}_{\mathrm{s}}^{(0)}}{\partial t} \cdot \mathbf{T} \nabla_{x} c^{(0)}=0 .
\end{aligned}
$$




\section{Conclusions and perspectives}

Homegenisation of the pore-scale equations that describe advective-diffusive solute transport in an elastic porous medium leads to Biot's model of consolidation (Eqs. 13-16) and to transport equation (Eq. 29). This latter can be expressed in a simpler manner as follows

$$
\phi \frac{\partial c}{\partial t}+\phi\left(\vec{V}_{\mathrm{f}}-\vec{V}_{\mathrm{s}}\right) \cdot \nabla c+\vec{V}_{\mathrm{s}} \cdot \mathbf{T} \nabla c=\nabla \cdot\left(\mathbf{D}^{\mathrm{eff}} \nabla c\right),
$$

where $\vec{V}_{\mathrm{f}}$ and $\vec{V}_{\mathrm{s}}$ denote the fluid and solid intrinsic macroscopic velocities, respectively.

Besides the usual accumulation, advection and effective diffusion terms, the coupling term $\vec{V}_{\mathrm{S}} \cdot \mathbf{T} \nabla c$ is emerging from the scale transition. This term is associated with the no-slip boundary condition at the fluid-solid interface and characterizes a mechanically-induced solute transport mechanism. It can be seen as a convective transport in the boundary layer moving at the solid phase velocity. It is weighted by the tortuosity tensor $\mathbf{T}$ which represents a geometrical property of the unit cell micro-structure; for isotropic porous media it comes down to a scalar ranging from 0 to 1 . An important feature of this coupling term is that it occurs only whenever there exits macroscopic advection: it does not appear in the case of a purely diffusive solute transport.

The influence of this additional term should be negligible in classical cases of consolidating porous media. Nevertheless, when focusing on solute transport in porous media submitted to cyclic loading, the cumulative effect of this mechanically-induced transport mechanism may become predominant. Experimental evidence of this term may be tricky to bear out. However, a numerical approach would bring some interesting features. The above developments have been done with specific orders of the dimensionless numbers $\mathbb{F}_{\mathrm{L}}, \mathbb{N}_{\mathrm{L}}$ and $\mathbb{P} e_{\mathrm{L}}$. Future works will include the analysis of different cases and particularly for higher Péclet number where dispersive effects come up.

\section{References}

N.A. Alshawabkeh, N. Rahbar, T. Sheahan, A model fo contaminant mass flux in capped sediment under consolidation, Journal of Contaminant Hydrology 78 (2005) 147-165.

F. Arega, E. Hayter, Coupled consolidation and contaminant transport model for simulating migration of contaminants through the sediment and a cap, Applied Mathematical Modelling 32 (2008) 2413-2428.

J.L. Auriault, E. Sanchez-Palencia, Etude du comportement macroscopique d'un milieu poreux saturé déformable, Journal de Mécanique 16 (1977) 37-53.

J.L. Auriault, Heterogeneous medium: is an equivalent macroscopic description possible ?, International Journal of Engineering Science 29 (1991) 785-795. 
J.L. Auriault, P. Adler, Taylor Dispersion in porous media: analysis by multiple scale expansions, Advances in Water Resources 18 (1995) 217-226.

J.M. Brannon, M.E Pointdexter-Rollings, Consolidation and contaminant migration in a capped dredged material deposit, The Science of Total Environement 91 (1990) 115-126.

L. Dormieux, E. Lemarchand, Modélisation macroscopique du transport diffusif, Oil and Gas Science and Technology, Revue IFP, 55 (2000) 15-34.

R.C. Evans, T.M. Quinn, Dynamic Compression Augments Interstitial Transport of a Glucose-Like Solute in Articular Cartilage, Biophysical Journal 91 (2006) 1541-1547.

P.J. Fox, Coupled large strain consolidation and solute transport. I: Model development, Journal of Geotechnical and Geoenvironmental Engineering 133 (2007) 3-15.

G.H. Goldsztein, J.C. Santamarina, Solute transport during cyclic flow in saturated porous media, Applied Physics Letters 85 (2004) 2432-2434.

R.T. Mauck, C.T. Hung, G.A. Ateshian, Modelling of neutral solute transport in a dynamically loaded porous permeable gel: implications for articular cartilage biosynthesis and tissue engineering, Journal of Biomechanical Engineering 125 (2003) 602-614.

B.P. O'Hara, J. Urban, A. Maroudas, Influence of cyclic loading on the nutrition of articular cartilage, Ann Rheum Dis. 49 (1990) 536-539.

G.P. Peters, D.W. Smith, Solute transport through a deforming porous medium, International Journal for Numerical and Analytical Methods in Geomechanics 26 (2002) 683-717.

M. Quintard, Diffusion in isotropic and anisotropic porous systems: Threedimensional calculations, Transport in Porous Media 11 (1993) 187-199.

E. Sanchez-Palencia (1980) Non Homogeneous Media and Vibration Theory, Lecture Notes in Physics, Springer-Verlag, New-York.

B.G. Sengers, C.W.J. Oomens, F.P.T. Baaijens, An integrated finite-element approach to mechanics, transport and biosynthesis in tissue engineering, Journal of Biomechanical Engineering 126 (2004) 82-91.

D.W. Smith, One-dimensional contaminant transport through a deforming porous medium: theory and a solution for a quasi-steady-state problem, International Journal for Numerical and Analytical Methods in Geomechanics 24 (2000) 693-722.

M.L.K. Tate, Mixing Mechanisms and Net Solute Transport in Bone, Annals of Biomedical Engineering (2001) 810-811. 
F. Urciuolo, G. Imparato, and P.A. Netti, Effect of Dynamic Loading on Solute Transport in Soft Gels Implication for Drug Delivery, AIChE Journal 54 (2008) 824-834.

L. Wang, S.C. Cowin, S. Weinbaum, S.P. Fritton, Modeling Tracer Transport in an Osteon under Cyclic Loading, Annals of Biomedical Engineering 28 (2000) $1200-1209$. 